\title{
Independência do Banco Central e Equilíbrio Fiscal: algumas observações para o caso brasileiro
}

\author{
HELDER FERREIRA DE MENDONÇA*
}

Central Bank Independence and Fiscal Equilibrium: some observations for the Brazilian case. The objective of this paper is to analyze the main theoretical arguments for the analysis of the conduction of monetary policy on the fiscal side. Besides this, an analysis is made of the possible effects on the fiscal balance from the conduction of the monetary policy in the search for price stability after the Real Plan and due to an increase in the central bank independence (CBI) in the Brazilian case. The findings denote that the strategy for the conduction of the adopted monetary policy and the increase in the degree of $\mathrm{CBI}$ did not contribute to an imrovement in the fiscal balance.

Key words: central bank independence; inflation; public debt.

JEL Classification: E58; E63.

\section{INTRODUÇÃO}

Com o objetivo de aumentar a credibilidade na condução da política monetária e neutralizar os problemas potenciais oriundos do caso de dominância fiscal, a proposição de independência do banco central (IBC) tem recebido apreciável atenção tanto por teóricos quanto por responsáveis pela política monetária. As principais modificações na legislação de bancos centrais para torná-los mais independentes podem ser sumariadas em três pontos básicos (Posen, 1998): (i) capacidade do banco central em rejeitar a monetização da dívida pública; (ii) garantia de estabilidade para o mandato do presidente do BC; e (iii) prioridade de objetivo para a estabilidade de preços. 0 primeiro ponto está associado ao argu-

* Professor do Departamento de Economia da Universidade Federal Fluminense e pesquisador do CN Pq. E-mail: helderfm@hotmail.com. Submetido: A gosto 2003; aceito: A bril 2004. 
mento dominante na literatura de que um dos principais elementos responsáveis pela inflação de longo termo é o uso da receita de senhoriagem pelo governo. Destarte, a adoção de um banco central independente $(\mathrm{BCl})$ eliminaria a passividade monetária, e, portanto, o governo teria que ser mais responsável com seus gastos, pois estaria limitado à sua restrição orçamentária. ${ }^{1}$

Em conformidade com o que foi exposto acima, Tabellini (1986) afirma que a IBC é capaz de afetar o tamanho do déficit orçamentário. Esta proposição é ratificada por evidências empíricas que mostram a existência de uma relação negativa entre a IBC e o déficit. ${ }^{2} \mathrm{~N}$ ão obstante, é importante sal ientar que a maioria dos estudos leva em conta apenas o caso de países industrializados. R ecentemente, Sikken \& de H aan (1998), utilizando vários índices de IBC, analisaram a relação entre a IBC e o déficit orçamentário para um grupo de trinta países em desenvolvimento. A principal conclusão que chegaram foi a de que não existe a relação sobredita. A demais, diversos estudos empíricos não apresentam resultados que comprovem uma estreita relação entre monetização e déficit (vide tabela 1).

Tabela 1: Estudos empíricos sobre a monetização de déficits orçamentários

\begin{tabular}{llll}
\hline \multicolumn{1}{c}{ Estudo } & \multicolumn{1}{c}{ № de países } & Período & Conclusões \\
\hline Dombusch \& Fischer (1981) & 7 & $1960-77$ & Há evidência em 3 casos \\
Giannaros \& Kolluri (1985) & 10 industrializados & $1950-81$ & Sem evidência \\
Protopapadakis \& Siegel (1987) & 10 industrializados & $1952-83$ & Sem evidência \\
Demopoulus, Katsimbris \& Milles (1987) & 8 industrializados & $1961-80$ & Alguma evidência \\
Bamhart \& Darrat (1988) & 7 industrializados & $1960-84$ & Sem evidência \\
Burdekin \& Laney (1988) & 12 industrializados & $1960-83$ & Alguma evidência \\
de Haan \& Zelhorst (1990) & 17 desenvolvimento & $1961-85$ & Evidência limitada \\
Burdekin \& Wohar (1990) & 8 industrializados & $1962-85$ & Alguma evidência \\
Karras (1994) & 19 industrializados & $1949-89$ & Sem evidência \\
& e 13 desenvolvimento & & \\
Brown \& Yousefi (1996) & 10 desenvolvimento & $1950-89$ & Sem relação causal \\
& & & entre déficit e inflação \\
Sikken \& de Haan (1998) & 30 desenvolvimento & $1973-94$ & Evidência limitada \\
Posen (1998) & 68 & Décadas: & Sem evidência \\
& & $50-80$ & \\
\hline
\end{tabular}

Um outro ponto que merece atenção quando se avalia a conseqüência da adoção de um $\mathrm{BCI}$ para o lado fiscal refere-se ao fato de que a busca da estabilidade de preços pode promover uma elevação na taxa real de juros. Logo, o efeito de uma política monetária contracionista poderia implicar a necessidade da

\footnotetext{
${ }^{1}$ Para uma análise sobre as mudanças na condução da política monetária na América Latina, ver O rtiz (2002).

${ }^{2}$ Ver, M asciandaro \& Tabellini (1988), Grilli, M asciandaro \& Tabellini (1991), e Haan \& Sturm (1992).
} 
geração de superávits primários para pagar os custos do acréscimo da dívida enquanto a credibilidade está sendo construída (King, 1995). ${ }^{3}$

0 presente trabalho tem como objetivo analisar, de forma simplificada, os principais argumentos teóricos para a análise dos efeitos da condução da política monetária sobre o lado fiscal. A demais, é feita uma análise para o caso brasileiro referente ao período posterior à introdução do Plano R eal avaliando a possível influência do aumento da IBC sobre o equilíbrio fiscal. Além desta introdução, 0 artigo encontra-se dividido em mais três seções. A segunda seção apresenta os principais argumentos teóricos que mostram uma conexão entre as políticas fiscal e monetária; a terceira seção faz uma avaliação do caso brasileiro; por último, é apresentada a conclusão do artigo.

\section{A CONEXÃO ENTRE AS POLÍTICAS MONETÁRIA E FISCAL}

0 principal elo entre as políticas monetária e fiscal refere-se à restrição orçamentária do governo. No caso de um aumento do déficit público é provável que ocorra uma el evação nos juros pagos sobre os títulos emitidos pelo governo ou na base monetária para que seja possível obter uma forma de financiamento. $N$ esse sentido, uma maior disciplina fiscal seria capaz de reduzir a taxa de juros incidente sobre 0 estoque da dívida. A demais, governos que contam com a receita de senhoriagem ou que possuem dificuldades no acesso ao mercado de crédito para financiar seus gastos, têm como grande benefício proveniente da redução do déficit, a maior facilidade para a busca da estabilidade de preços. Entrementes, no caso de existir a possibilidade do governo emitir novos títulos, pode não haver a conexão entre o tamanho do déficit e a receita de senhoriagem (Taylor, 1995).

A interação entre as políticas monetária e fiscal revela que a estratégia adotada pelo responsável da política monetária, na busca da estabilidade de preços, pode afetar a capacidade do governo no financiamento do déficit. Por outro lado, a necessidade de financiamento do setor público pode representar um limite à independência operacional da autoridade monetária. De acordo com os resultados obtidos por Fry (1998), o tamanho do déficit do governo e a forma como ele é financiado determina a IBC no caso de países em desenvolvimento. A lém disso, alguns autores como Calvo (1988) defendem a idéia de que a tendência de o banco central inflacionar a economia é uma função da dívida pública, e que, portanto, uma redução de seu estoque seria capaz de reduzir a pressão sobre o banco central implicando maior credibilidade à condução da política monetária. ${ }^{4}$ Sikken $\&$ de $\mathrm{H}$ aan (1998) destacam pelo menos quatro canais em que o aumento da dí-

\footnotetext{
${ }^{3}$ Este ponto será melhor analisado no fim da próxima seção.

${ }^{4}$ Durante os anos 80 , diversos trabalhos procuraram analisar o efeito do déficit e da dívida sobre a credibilidade da política monetária tendo como base a estrutura teórica desenvolvida por Kydland \& Prescott (1977). Para o leitor interessado na literatura, ver Lucas \& Stokey (1983), Persson, Persson \& Svensson (1987), Calvo (1988), e Bohn (1988).
} 
vida pode levar a uma expansão monetária: (i) pressões políticas para estabilizar a taxa de juros; (ii) problema de inconsistência temporal da política monetária; (iii) a teoria da senhoriagem ótima; e (iv) a hipótese de dominância fiscal.

0 primeiro canal diz respeito ao fato de que, em geral, o elo entre as políticas monetária e fiscal é analisado em um ambiente no qual a autoridade monetária é obrigada a estabilizar a taxa de juros. É importante ressaltar que apesar de a taxa de juros representar o principal instrumento para a ação da política monetária, uma volatilidade el evada implica aumento da incerteza acarretando efeitos indesejáveis para a economia (queda do investimento e produto, aumento da taxa de desemprego etc.). Assim, se um aumento na dívida pública levar a um incremento da taxa de juros, o banco central é compelido a monetizar o déficit. ${ }^{5}$

Para que se possa compreender melhor a idéia presente no segundo e terceiro canal supradito, considere, a seguir, a equação que representa a restrição orçamentária intertemporal do governo:

(1) $\dot{B}=D_{t}+i_{t} B_{t}-\dot{M}_{t}$,

onde: $\mathrm{B}=$ montante da dívida governamental; $\mathrm{D}=$ déficit primário; $\mathrm{i}=$ taxa de juros; $\mathrm{M}$ = base monetária; que expressa em termos reais, corresponde a

(2) $\dot{b}_{t}=d_{t}+i_{t}\left[i_{t}-\left(\dot{p}_{t} / p_{t}\right)\right] b_{1}-\dot{M}_{t} / p_{t}$,

onde: $\mathrm{p}=$ deflator; $\mathrm{b}=\mathrm{B} / \mathrm{p}$; .

A equação acima permite observar que o estoque da dívida pode ser reduzido de três formas: (a) via redução do déficit primário; (b) por meio de ganho de capital do governo no caso de a taxa de inflação atual exceder a inflação que compõe a taxa de juros; e (c) via senhoriagem.

O s dois últimos pontos são utilizados com freqüência na literatura para a análise entre as políticas monetária e fiscal. 0 segundo ponto (b) está relacionado à idéia de inconsistência temporal da política monetária. Sob esta perspectiva, o público determina a taxa esperada de inflação e a autoridade monetária determina a taxa atual de inflação. N essa estrutura, os governos são tentados a causar uma inflação surpresa como forma de reduzir o encargo real da dívida (sobretudo no caso de taxas de juros nominais de longo prazo fixas). 0 terceiro ponto (c) pode ser mais bem compreendido fazendo-se uso da equação,

$$
S_{t}=M_{t} / P_{t}=\left(M_{t} / M_{t}\right)\left(\dot{M}_{t} / p_{t}\right)=\alpha \cdot m,
$$

onde: $a=$ taxa de expansão monetária; $m$ = estoque real de moeda.

Dado que em países em desenvolvimento há uma baixa capacidade de tributação do produto (PIB), a senhoriagem torna-se um instrumento factível para financiar os gastos do governo. N ão obstante, Sikken \& de Haan (1998) fazem três considerações em relação a essa possibilidade:

(i) o uso permanente da expansão monetária deve reduzir a receita de senho-

\footnotetext{
${ }^{5}$ Esta última observação representa o principal argumento para a adoção de um $\mathrm{BCl}$ como estratégia para evitar a ocorrência do fenômeno em questão.
} 
riagem (uma taxa de inflação el evada aumenta as expectativas inflacionárias e reduz a base $-\mathrm{m}-$ mais do que 0 aumento em a);

(ii) diversos países em desenvolvimento utilizam um regime de câmbio no qual a moeda doméstica é atrelada a uma moeda com credibilidade elevada, e, por conseguinte, não têm o poder de determinar a expansão monetária da economia; e

(iii) o uso do imposto inflacionário implica perda do poder de compra do público; logo, maximizar receita de senhoriagem pode não ser adequado do ponto de vista social.

O quarto canal entre o aumento da dívida e a expansão monetária refere-se à hipótese de dominância fiscal (Sargent \& Wallace, 1981). Sob esta interpretação, a autoridade fiscal define os déficits e superávits no presente e no futuro sem consultar a autoridade monetária, e, portanto, determina o montante de receita provinda da venda de títulos e senhoriagem. $N$ este caso, a autoridade monetária diante de uma restrição imposta pela demanda por títulos do governo tende a financiar, via senhoriagem, o restante da receita necessária para satisfazer a demanda da autoridade fiscal. Sob esta perspectiva, há a tendência de que o responsável pela política não mantenha o equilíbrio fiscal devido à expectativa de que o banco central garantirá os recursos necessários para satisfazer os gastos realizados. Assim sendo, o provável resultado de uma dominância fiscal é o aumento do déficit em decorrência da el evação dos gastos e da taxa de inflação proveniente da emissão monetária pelo banco central. ${ }^{6}$

A inda sob a interpretação de Sargent \& Wallace (1981), um outro ponto a ser considerado diz respeito ao caso em que há um elevado estoque da dívida e déficit público capaz de provocar um aumento da taxa real de juros acima da taxa de crescimento da economia. $N$ esse caso, a tentativa da política monetária em reduzir a taxa de inflação pode ter um efeito perverso sobre a economia. A lógica para este fenômeno refere-se ao fato de que uma contração monetária hoje para reduzir a taxa de inflação provoca um aumento na relação dívida/PIB, que devido à necessidade de pagamentos de juros mais elevados e ao tamanho do déficit implicam aumento da base monetária no futuro. N este caso, conforme proposto por Taylor (1995), uma redução da taxa de juros para níveis inferiores à taxa de crescimento da economia poderia restaurar a capacidade da política monetária no controle da inflação.

O problema da dominância fiscal tem sido considerado um dos principais argumentos teóricos a favor da adoção de um $\mathrm{BCl}$. A visão tradicional para a análise entre as políticas fiscal e monetária tem como elemento central a idéia de que a autoridade fiscal define o déficit independente da autoridade monetária, e que

\footnotetext{
${ }^{6}$ Conforme destacado por Sargent \& Wallace (1981), o ponto fundamental para avaliar se a autoridade monetária será capaz de controlar de forma permanente a inflação concentra-se na demanda por títulos do governo. No caso de a demanda por título implicar uma taxa de juros que remunere os títulos acima da taxa de crescimento da economia, e a autoridade fiscal incorrer em déficits, a autoridade monetária perde a capacidade de definir a taxa de inflação.
} 
esta última é forçada a emitir moeda para satisfazer as necessidades de financiamento do governo. A pesar de o argumento oferecer uma consistência interna apreciável, alguns autores têm ressaltado que esse tipo de suposição não é coerente com o mundo real. King (1995, p. 171) apresenta dois motivos para essa visão:

"First, seigniorage - financing the deficit by issuing currency rather than bonds - is very small relative to other sources of revenue. Second, over the past decade or so, governments have become increasingly the power over monetary policy to an independent central bank with the specific objective of price stability."

U ma conseqüência do segundo ponto citado é que o esforço para reduzir a inflação acarreta uma taxa menor que a esperada, o que por sua vez, gera um problema de ordem fiscal. A introdução de uma estratégia para a política monetária que diminua a taxa de inflação, mas que não possui total credibilidade, acarreta uma elevação da taxa real de juros sobre a dívida pública. ${ }^{7}$ Portanto, enquanto a credibilidade é construída, há a necessidade de uma receita adicional para financiar o maior custo da dívida. Assim, emerge o problema que King (1995) denominou como "desagradável aritmética fiscal". ${ }^{8}$

0 principal aspecto a ser realçado consiste no fato de que a inflação esperada declinará mais lentamente que a inflação atual. Assim, o êxito em desinflacionar a economia reduz a taxa de crescimento do PIB (devido ao aumento da taxa real de juros), mas não reduz a despesa com juros relativa ao estoque da dívida até que a nova política conquiste credibilidade. Além disso, o nível de superávit primário consistente com a relação dívida/PIB aumenta no curto prazo por um montante proporcional à razão dívida/PIB inicial e a redução na taxa de inflação média. N o longo prazo, o superávit primário pode retornar ao nível inicial quando as expectativas inflacionárias tiverem se ajustado. Entretanto, países com histórico de dificuldade no controle da inflação devem levar mais tempo para obter este resultado (King, 1995).

\footnotetext{
${ }^{7}$ A justificativa para o aumento da taxa real de juros representa o caso inverso ao apresentado no item b (ganho de capital do governo no caso de a taxa de inflação atual exceder a inflação que compõe a taxa de juros).

${ }^{8} \mathrm{O}$ esquema 1 apresenta um paralelo entre a idéia básica sobre a desagradável aritmética monetarista e a desagradável aritmética fiscal.

${ }^{9} \mathrm{O}$ exemplo a seguir (King, 1995) mostra, na prática, de que forma se manifesta o problema. Suponha uma dívida/PIB de $50 \%$ estável, e que a mudança na política monetária reduziu a média da inflação de 6 para $2 \%$ a.a. Assim, para garantir uma taxa constante da dívida/PIB há a necessidade de um salto no superávit primário de no mínimo $2 \%$ do PIB. Se as expectativas em relação ao novo regime se ajustarem após cinco anos, então o aumento no superávit primário deveria ainda ser de 1\% do PIB após cinco anos.
} 
Esquema 1: Desagradável aritmética monetarista e fiscal

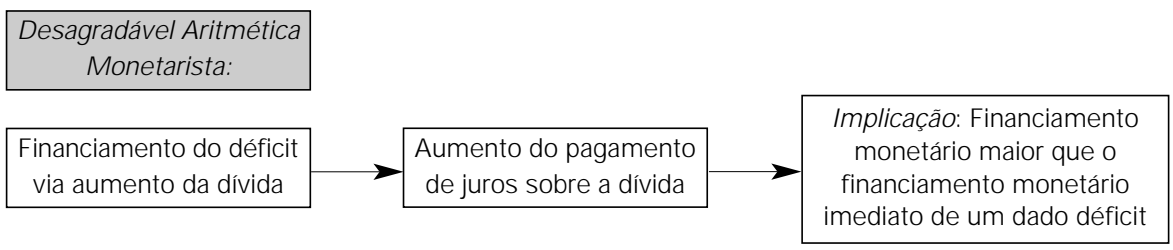

\section{Desagradável Aritmética \\ Fiscal:}

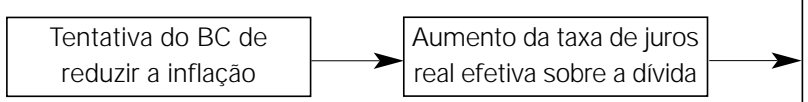

Implicação: Necessidade de superávit primário adicional para financiar o aumento do custo da dívida enquanto a credibilidade é construída

\section{ALGUM AS OBSERVAÇÕES PARA O CASO BRASILEIRO}

Um ponto importante a ser observado consiste em verificar se o sucesso no controle da inflação no período posterior à introdução do Plano Real foi capaz de reduzir (ou aumentar) a taxa de juros implicando menor (ou maior) pressão sobre o endividamento público. Conforme pode ser observado por meio da figura 1 verifica-se que apesar de ter ocorrido uma queda significativa na taxa real de juros ao longo do período, ela se situou acima da taxa de crescimento da economia na maior parte do tempo.

Figura 1

Taxa de crescimento do PIB e taxa real de juros

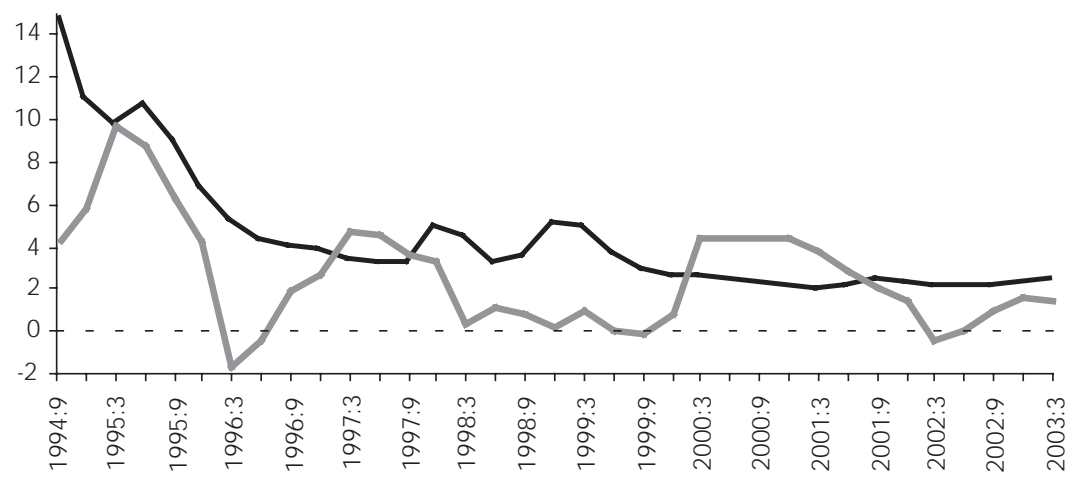

SELIC real trim

PIB - taxa acumulada até o trimestre (\%) 
N ota: SELIC deflacionada pelo IPCA (jul/1994=100). As taxas trimestrais são calculadas elevando-se a média geométrica das taxas mensais a 3 (meses).

A constatação acima remete ao problema ressaltado por Sargent \& Wallace relativo à possibilidade de perda de controle da autoridade monetária sobre a inflação. $N$ esse sentido, poderia ser iniciado um ciclo marcado por aumento da taxa de juros (ou manutenção no caso de se encontrar em um patamar muito elevado) como tentativa de o banco central conter a pressão inflacionária e a queda na taxa de crescimento econômico devido à redução no consumo e no investimento. Logo, a combinação desses dois fatos teria como resultado um incremento no endividamento público.

$\mathrm{N}$ a prática, observa-se que a preocupação do Banco Central do Brasil (BCB) em evitar um desequilíbrio externo devido às flutuações no cenário internacional - sobretudo em função dos choques asiático (1997) e russo $(1998)^{10}$ - fez com que a taxa de juros básica da economia (SELIC) sofresse fortes variações que culminaram com o aumento das necessidades de financiamento do setor público com juros reais. Deve-se ressaltar que a estratégia de combate à inflação que vigorou até janeiro de 1999 (baseada em variantes do regime de câmbio fixo) impedia uma ação efetiva da política monetária no controle da taxa de juros. Além disso, é importante notar que embora as el evações observadas na SELIC nos anos de 1997 e 1998 terem focado o ajuste externo, o principal objetivo dos aumentos observados era evitar que ocorresse um ataque especulativo sobre a economia que forçasse uma desvalorização cambial, e que, por conseguinte, colocasse em risco a estabilidade de preços.

Com o fim do uso da taxa de câmbio como principal estratégia de combate à inflação (janeiro de 1999) houve a necessidade da busca de uma nova âncora nominal. Em junho de 1999 foi implantado um novo regime monetário no Brasil com base na utilização de metas para a inflação. ${ }^{11} \mathrm{~N}$ esse novo modelo para a condução da política econômica, tornou-se explícito o uso da taxa de juros de curto prazo como principal instrumento para o alcance da meta de inflação anunciada. A conseqüência de um cenário em que a taxa de juros encontrava-se acima da taxa de crescimento da economia combinado à el evação do endividamento público devido à forte desvalorização da moeda, tornou necessária a geração de superávits primários como tentativa de alcançar o equilíbrio fiscal (vide figura 2).

\footnotetext{
${ }^{10}$ Durante o período sob análise a balança de transações correntes encontrava-se deficitária, logo o principal mecanismo utilizado (após a forte entrada de recursos provinda do processo de privatizações) consistiu na elevação da taxa de juros como mecanismo para tornar a balança de capitais superavitária e equilibrar o balanço de pagamentos.

${ }^{11}$ Para uma análise da teoria do regime de metas de inflação e do seu funcionamento no Brasil, ver M endonça (2001 e 2002), e N eto (1999).
} 
Figura 2

Dívida pública e superávit primário

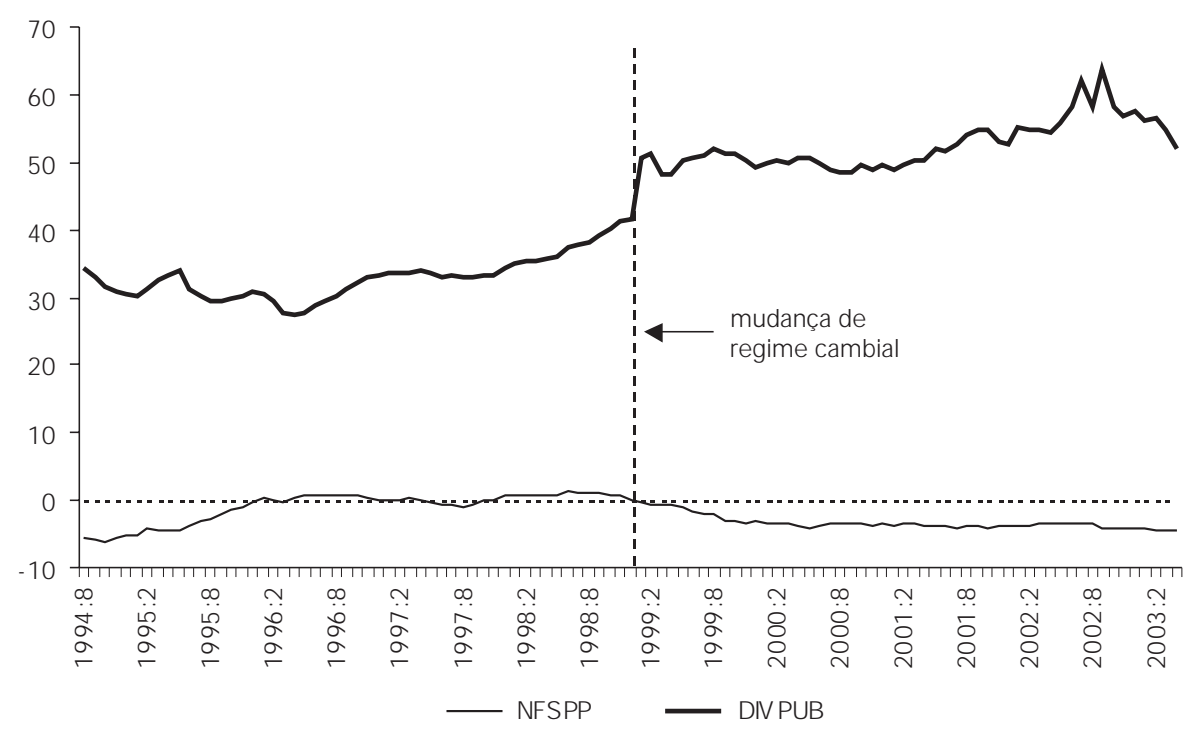

Nota: NFSPP — Necessidades de financiamento - setor público - primário — acum. 12 meses (\% PIB); DIVPUB — Dívida - total — setor público - líquida (\% PIB)

As observações acima permitem conjeturar que o Brasil no período pós-R eal representa um caso de desagradável aritmética fiscal. A introdução do Plano Real em 1994 foi responsável por uma mudança qualitativa do ponto de vista do controle inflacionário. A pesar de a taxa de inflação ter se mantido relativamente estável após a implementação do Plano, a estratégia utilizada para o combate à inflação sofreu mudanças significativas ao longo dos anos. ${ }^{12} \mathrm{As}$ alterações ocorridas na condução da política monetária não permitiram que fosse construída uma credibilidade suficiente para que as políticas anunciadas ficassem imunes aos choques sobre a economia. D estarte, o caso brasileiro enquadra-se na situação em que a credibilidade ainda está sendo construída.

U m importante elemento para análise refere-se ao comportamento do $\mathrm{BC} B$ após a introdução do Plano sobredito. A preocupação da autoridade monetária em assegurar o compromisso com a estabilidade de preços fez com que o índice de independência real do BCB aumentasse de forma significativa (R igolon, 1997). ${ }^{13}$ Dessa forma, é importante verificar se o incremento na independência do BCB, observado nos últimos anos, está associado a alguma mudança na evolução do

\footnotetext{
${ }^{12} \mathrm{~A}$ s principais mudanças ocorridas se referem à alteração do regime de câmbio em janeiro de $1999 \mathrm{e}$ à adoção do regime de metas de inflação em junho do mesmo ano.

${ }^{13}$ Para o leitor interessado em uma resenha da literatura sobre IBC que contém a explicação detalhada dos índices de independência real e legal, ver Cukierman (1996). 0 apêndice apresenta a metodologia para a mensuração do grau de independência do BCB de 1986 até 2002.
} 
quadro fiscal brasileiro. Esta observação é importante porque alguns economistas que analisam a proposição de IBC sugerem que a presença de um $\mathrm{BCI}$ é capaz de levar a uma combinação de inflação baixa com menores déficits ficais para a economia (Goodhart, 1995). A idéia básica consiste no fato de que um BCI evitaria o uso da receita de senhoriagem, o que, por sua vez, poderia acarretar uma contenção nos gastos do governo.

Com o objetivo de avaliar o possível efeito de um aumento da independência do BCB sobre o quadro fiscal brasileiro foi feita uma análise com base em alguns resultados empíricos. Utilizou-se o método dos mínimos quadrados para avaliar a influência do grau de independência (GIBC) sobre duas variáveis: resultado primário (N FSPP - necessidades de financiamento do setor público \% PIB - primário) e necessidades de financiamento do setor público - juros reais (N FSPJR - \% PIB). Para tanto, foram utilizados dados anuais para o período compreendido entre 1986 e 2002. D evido à mudança estrutural ocorrida com a introdução do Plano Real em 1994 foi introduzida uma variável dummy na forma aditiva ( $D=0$ antes de 1994 e $D=1$ a partir de 1994) nas estimações.

A justificativa para a seleção das variáveis sobreditas pode ser compreendida da seguinte forma. É esperado que um aumento da IBC esteja relacionado a uma redução nos gastos do governo. 0 gráfico de dispersão (grau de independência do BCB X N FSPP) localizado no lado esquerdo da figura 4 sugere que essa perspectiva é correta, uma vez que há uma correlação negativa entre as séries que corresponde a 31\%; 0 efeito de um aumento da independência do BC sobre a despesa do setor público com o pagamento de juros não é evidente. Uma maior independência pode significar uma política monetária mais restritiva que culmina com a elevação da N FSPJ R; por outro lado, o sucesso no combate à inflação significa conquista de credibilidade contribuindo para uma menor taxa de juros. O gráfico localizado no lado direito da figura 3 não contribui para dirimir essa dúvida, pois a correlação entre as séries é quase nula.

Figura 3
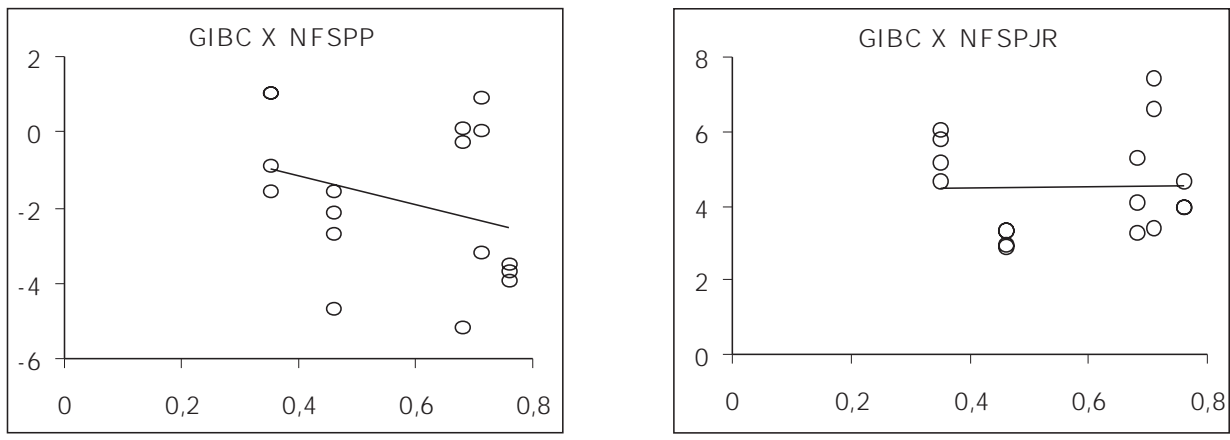
0 resultado obtido com as estimações para o período de 1986/2002 (vide tabela 2) indica que o grau de independência tem sinal negativo tanto para a N FSPP quanto para a N FSPJ R, ao passo que a dummy apresentou sinais positivos. Além disso, a hipótese nula de ausência de efeito é rej eitada para ambas as variáveis ao nível de significância de $5 \%$. Este resultado indica que as duas variáveis apresentam efeitos estatisticamente significativos sobre a NFSPP e a N FSPJ R, com probabilidade de erro de $5 \%$. N ão obstante, o coeficiente de determinação denota uma explicação de apenas 35\% da variação da N FSPP e 32\% da variação da N FSPJ R. Em outras palavras, os resultados encontrados sugerem que um aumento da IBC contribuiu para uma melhora do resultado primário e uma menor despesa com o pagamento de juros reais, entretanto, o efeito não é forte o suficiente para se esperar uma melhora no quadro fiscal.

Tabela 2: Estimações (MQO)

\begin{tabular}{lccccccc}
\hline $\mathrm{n}=17$ & constante & estat.- $\mathrm{t}$ & GIBC & estat.- t & Dummy & estat.- $\mathrm{r}$ & $\mathrm{R}^{2}$ \\
\hline NFSPP & $\mathbf{8 , 7 5 9 0}$ & $2,2280^{*}$ & $-25,2194$ & $-2,6316^{*}$ & 7,2123 & $2,3207^{*}$ & 0,3487 \\
NFSPJ R & 10,6243 & $3,9999^{*}$ & $-15,6747$ & $-2,4208^{*}$ & 5,3515 & $2,5486 *$ & 0,3173 \\
\hline
\end{tabular}

Nota: * Significância a $5 \%$.

\section{CONSIDERAÇÕES FIN AIS}

A análise apresentada na seção anterior sugere que apesar de ter havido um aumento do grau de IBC do BCB ao longo do período analisado, não se pode esperar que ocorra uma melhora no quadro fiscal. A idéia de que um $\mathrm{BCI}$ contribuiria para a redução das despesas com o pagamento de juros e maior equilíbrio no resultado primário não pode ser descartada. Entretanto, conforme pode ser observado por meio da figura 4, o aumento da IBC do BCB ao longo do tempo não foi suficiente para conter a elevação no estoque do endividamento público. A deterioração do quadro fiscal com a estabilidade de preços é explicada por dois motivos básicos: (i) com a estratégia da condução da política monetária voltada para a busca da estabilidade de preços, o Tesouro $\mathrm{N}$ acional ficou impedido de monetizar parte da dívida (desde 1995 a receita de senhoriagem é inferior a 1\% do PIB); e (ii) a estrutura de indexação da dívida pública atrelada em grande medida à SELIC. 
Figura 4

Dívida pública - IBC - indexador (SELIC)

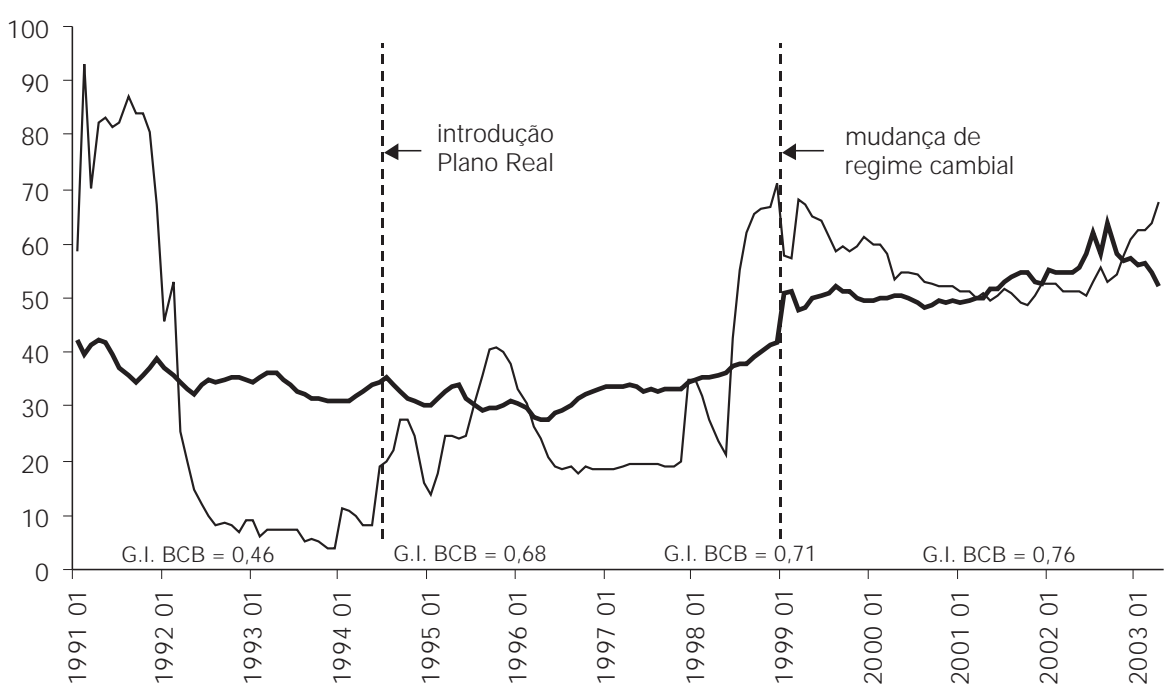

Títulos fed. index. SELIC (\%) _ Dívida líq. (\% PIB)

O segundo ponto supracitado merece uma maior atenção, pois representa a possibilidade de que ao invés de benefícios para o equilíbrio fiscal, um aumento na dominância monetária implique efeitos perversos sobre a economia. Uma das principais razões para a elevação do estoque da dívida pública se deve ao fato de a SELIC ser o principal indexador dos títulos públicos. ${ }^{14} \mathrm{~N}$ esse sentido, a manutenção da alta taxa real de juros praticada no período pós-R eal, com o objetivo de manter a inflação sob controle, representou uma fonte para o comportamento da dívida no período. Soma-se a isto o fato de que o câmbio, segundo indexador mais importante para os títulos públicos, contribuiu de forma decisiva para o salto da dívida observado no período posterior à mudança do regime cambial. Um outro ponto a ser ressaltado se refere ao fato de a economia apresentar um crescimento econômico incapaz de promover o equilíbrio macroeconômico. Logo, o receituário mais óbvio para neutralizar as mazelas de um desequilíbrio fiscal tem se concentrado na necessidade da geração de superávits primários com o objetivo de evitar uma aceleração na trajetória dívida/PIB. Portanto, o superávit primário observado no período recente não é um resultado inequívoco do aumento da IBC do $B C B$, mas sim uma necessidade oriunda do próprio cenário econômico.

Para evitar o problema acarretado pela dominância monetária recomendase a ação coordenada entre as autoridades monetária e fiscal de forma que a busca da estabilidade de preços combinada a uma política fiscal responsável não im-

${ }^{14}$ Vide figura 5 para observar a evolução da over-SELIC como indexador dos títulos públicos federais a partir de 1990. 
plique custos sociais desnecessários. Uma forma de atenuar o problema é via alteração da estrutura de indexação da dívida, reduzindo-se a proporção de títulos atrelados à SELIC e ao câmbio e aumentando a quantidade de títulos indexados à inflação. Deste modo, elevações na taxa de juros para neutralizar pressões inflacionárias levariam também a reduções na razão dívida/PIB aumentando a credibilidade da política antiinflacionária. ${ }^{15} \mathrm{O}$ principal ponto a ser realçado consiste no fato de que há a necessidade de que sejam levados em consideração os efeitos negativos que a ação de uma política monetária contracionista pode acarretar à política fiscal. Em outras palavras, é preciso que sejam desenvolvidos mecanismos que procurem eliminar não só os problemas provenientes da dominância fiscal, mas também aqueles oriundos da dominância monetária.

\section{REFERÊN CIAS BIBLIOGRÁFICAS}

BACK, J.L., M USGRAVE, R.A., (1941) "A Stable Purchasing Power Bond", A merican E conomic Review 31, p. 823-5.

BAR N HART, S.W. \& DARRAT, A.F. (1988) "Budget Deficits, M oney Growth and Causality: Further OECD Evidence", J ournal of International M oney and Finance, 7, 231-42.

BO H N , H . (1988) "W hy Do We H ave N ominal Government Debt?" , Journal of M onetary E conomics, 21, p. 127-40.

BROWN , K.H . \& YOUSEFI, M . (1996) “D eficits, inflation and central banks' independence: evidence from developing countries", A pplied E conomics L etters, 3, 505-9.

BURDEKIN, R.C. \& LANEY, L.O . (1988) "Fiscal Policymaking and the Central Bank Institutional Constraint", Kyklos, 41, 647-62.

BURDEKIN, R.C. \& W OHAR, M.E. (1990) “M onetary Institutions, Budget Deficits and Inflation. Empirical Results for Eight Countries", European Journal of Political Economy, 6, 531-51.

CALVO, G. (1988) "Servicing the Public Debt: The Role of Expectations", A merican Economic Review, 78, 647-61.

CUKIER M AN, A . (1996) "A Economia do Banco Central”, Revista Brasileira de Economia, Rio de Janeiro, 50(4), out./dez, 389-426.

de HAAN, J. \& STURM , J.E. (1992) "The Case for Central Bank Independence", Banca N azionale del Lavoro Q uaterly Review, 182, 305-27.

de HAAN, J. \& ZELHORST, D. (1990) "The impact government deficits on money growth in developing countries", J ournal of International M oney and Finance, 9, 455-69.

de M EN D ON ÇA, H.F. (2002) "R egimes M onetários e a Busca da Estabilidade de Preços: o Uso de M etas para a Taxa de Câmbio, Agregados M onetários e Inflação", R evista de E conomia Política, V. 22, N. 1 (85), jan-mar, p. 34-52.

. (2001) "M etas de Inflação: U ma Análise Preliminar para o Caso Brasileiro", Economia A plicada, FIPE/FEA-USP, São Paulo, V.5, N. 1, jan-mar, 129-58.

DEM O POULUS, G.D., KATSIM BRIS, G.M \& \& M ILER, S.M . (1987) “M onetary Policy and CentralBank Financing of Government Budget Deficits", European Economic Review, 31, 1023-50.

DORN BUSCH, R. \& FISCHER, S. (1981) "Budget Deficits and Inflation", in: Flanders, M .J \& \& Razin A. (eds), D evelopment in an Inflationary World, A cademic Press, N ew York, NY.

${ }^{15}$ Esse argumento foi construído originalmente por Back \& M usgrave (1941) sendo desenvolvido no período mais recente por Lucas \& Stokey (1983), Bohn (1988), Calvo (1988) e Calvo \& Guidotti (1990). 
FRY, M . (1998) "Assessing Central Bank Independence in Developing Countries: Do Actions Speak Louder Than Words?", O xford E conomic Papers: V. 50, N. 3, 512-29.

GIAN N AROS, D.S. \& KOLLURI, B.R. (1985) “D eficit Spending, M oney, and Inflation: some International Empirical Evidence", J ournal of M acroeconomics, 7, 401-17.

GOODHART, C. (1995) "Central Banking and Financial System", London: M acM illam.

GRILLI, V., M ASCIAN DARO, D. \& TABELLIN I, G. (1991) "Political and M onetary Institution and Public Financial Policies in the Industrial Countries", Economic Policy: a European Forum, 13, 341-92.

KARRAS, G. (1994) "M acroeconomic Effects of Budget Deficits: Further International Evidence", Journal of International M oney and Finance, 13, 190-210.

KIN G, M . (1995) "Commentary: M onetary Policy Implications of G reater Fiscal Discipline", in: Budget D eficits and D ebt: Issues and O ptions. Federal Reserve Bank of Kansas City. A ugustSeptember, 171-183.

KYDLAND, F. E. \& PRESCOTT, E. C. (1977) "Rules Rather than Discretion: the Inconsistency of O ptimal Plans", J ournal of Political Economic 85, N. 3, 473-92.

LUCAS, R. \& STOCKEY, N . (1983) "O ptimal Fiscal and M onetary Policy in an Economy Without Capital", J ournal of M onetary E conomics 12, 55-93.

MASCIANDARO, D.\& TABELLINI, G. (1988) "M onetary Regimes and Fiscal Deficits: a Comparative Analysis", in: H.-S. Cheng (ed.), M onetary Policy in Pacific Basin Countries, Kluwer A cademic Publishers, Boston, M A, 125-152.

N ETO , A. D. (1999) "Sobre as M etas inflacionárias", E conomia A plicada, FIPE/FEA-USP, São PauIo, V.3, N.3, 357-82.

ORTíZ, G. (2002) "M onetary Policy in a Changing Economic Environment: The Latin A merican Experience", in: Rethinking Stabilization Policy. Federal Reserve Bank of Kansas City. August, 207-39.

PERSSO N, M ., PERSSO N , T. \& SVEN SSO N, L. (1987) "Time Consistency of M onetary and Fiscal Policy", Econometrica 55, 1419-32.

POSEN, A . (1998) "Central Bank Independence and Disinflationary Credibility: A M issing Link", O xford E conomic Papers, V. 50, N. 3, 335-59.

PROTOPAPADAKIS, A.A. \& SIEGEL, J.J. (1987) “A re M oney Growth and Inflation R elated to Government Deficits? Evidence from Ten Industrialized Economies", J ournal of International M oney and Finance, 6, 31-48.

RIGOLON, F.J.Z. "Independência do Banco Central: Teoria e A plicações para o Brasil", Ensaios BNDES 3, abril 1997.

SARGENT, T.J. \& WALLACE, N . (1981) "Some Unpleasant M onetarist A rithmetic", Q uarterly Review, Federal Reserve Bank of $M$ inneapolis, Fall, 1-17.

SIKKEN , B.J. \& de HAAN , J. (1998) "Budget Deficits, M onetization, and Central Bank Independence in Developing Countries", O xford E conomic Papers. V. 50, N. 3, 493-511.

TABELLIN I, G. (1986) "M oney, D ebt, and D eficits in a Dynamic Game", J ournal of E conomic D ynamics and Control, V. 10, 427-42.

TAY LO R, J.B. (1995) "M onetary Policy Implications of Greater Fiscal Discipline", in: Budget D eficits and Debt: Issues and O ptions. Federal Reserve Bank of Kansas City. A ugust-September, $151-70$ 


\section{APÊNDICE: \\ $M$ etodologia para estimar 0 grau de independência do $B C B$}

Tabela A.1. Variáveis, pesos e códigos

\begin{tabular}{|c|c|c|c|}
\hline $\begin{array}{l}\text { Número } \\
\text { da variável }\end{array}$ & Descrição da variável & & códigos \\
\hline \multirow[t]{4}{*}{1} & Rotatividade do presidente do BC & & \\
\hline & Baixa rotatividade & & 1,0 \\
\hline & Média rotatividade & olp & 0,5 \\
\hline & Alta rotatividade & & 0,0 \\
\hline \multirow[t]{5}{*}{2} & Restrições ao financiamento do Setor Público & & \\
\hline & Alta & & 1,00 \\
\hline & Moderadamente alta & IIp & 0,66 \\
\hline & Moderadamente baixa & & 0,33 \\
\hline & Baixa ou inexistente & & 0,00 \\
\hline \multirow[t]{4}{*}{3} & Resolução de conflitos & & \\
\hline & Algumas resoluções a favor do banco & & 1,0 \\
\hline & Resolução a favor do governo em todos os casos & rc & 0,0 \\
\hline & Todos os outros casos & & 0,5 \\
\hline \multirow[t]{4}{*}{4} & Elaboração do orçamento do banco central & & \\
\hline & Maioria das vezes o banco central & & 1,0 \\
\hline & Banco central e Poder Executivo/Poder Legislativo & dcbb & 0,5 \\
\hline & Maioria das vezes Poder Executivo/Poder Legislativo & & 0,0 \\
\hline \multirow[t]{4}{*}{5} & Determinação dos salários e alocação dos lucros do banco central & & \\
\hline & Maioria das vezes pelo BC ou por lei & & 1,0 \\
\hline & Banco central e Poder Executivo/Poder Legislativo & wp & 0,5 \\
\hline & Maioria das vezes Poder Executivo/Poder Legislativo & & 0,0 \\
\hline \multirow[t]{5}{*}{6} & Metas para agregados monetários & & \\
\hline & Existência de metas; boa aderência & & 1,00 \\
\hline & Existência de metas; média aderência & qmst & 0,66 \\
\hline & Existência de metas; baixa aderência & & 0,33 \\
\hline & Não há metas & & 0,00 \\
\hline \multirow[t]{3}{*}{7} & Metas formais ou informais para as taxas de juros & & \\
\hline & Não & irt & 1 \\
\hline & $\operatorname{Sim}$ & & 0 \\
\hline \multirow[t]{6}{*}{8} & Prioridade dada para a estabilidade dos preços & & \\
\hline & Primeira prioridade é a estabilidade dos preços & & 1,00 \\
\hline & Primeira prioridade é a estabilidade da taxa de câmbio & & 0,66 \\
\hline & $\begin{array}{l}\text { Preços ou estabilidade da taxa de câmbio estão } \\
\text { entre os objetivos do BC, mas não representam }\end{array}$ & & \\
\hline & a primeira prioridade & pps & 0,33 \\
\hline & Nem preços ou câmbio estão entre os objetivos do BC & & 0,00 \\
\hline \multirow[t]{5}{*}{9} & BC funciona como Banco de Desenvolvimento? & & \\
\hline & Não & & 1,00 ` \\
\hline & Em alguma extensão & $\mathrm{fdb}$ & 0,66 \\
\hline & Sim & & 0,33 \\
\hline & O BC é fortemente envolvido na garantia de crédito subsidiado & & 0,00 \\
\hline
\end{tabular}

Fonte: Cukierman, Webb \& Neyapti (1992). 
Tabela A.2. Agregação

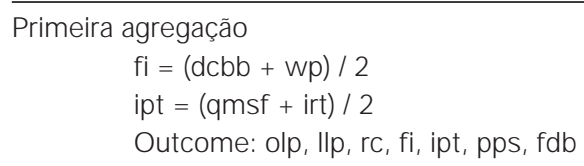

Segunda agregação

$$
\begin{aligned}
& A D I U=(o l p+l l p+r c+f i+i p t+p p s+f d b) / 7 \\
& A D I W=0,1 o l p+0,2 l l p+0,1 r c+0,1 f i+0,15 i p t+0,15 p p s+0,2 f d b
\end{aligned}
$$

Fonte: Cukierman, Webb \& Neyapti (1992).

OBS: ADIU - actual degree of independence unweighted;

ADIW - actual degree of independence weighted;

$\mathrm{fi}$ - financial independence;

ipt - intermediate policy targets.

Tabela A.3. Estimativas para o Brasil - 1986-2002

\begin{tabular}{lcccccc}
\hline Período & 1986 & $1987 / 89$ & $1990 / 92$ & $1994 / 96$ & $1997 / 99$ & $2000 / 02$ \\
\hline Variáveis & & & & & & \\
Olp & 0,00 & 0,00 & 0,00 & 0,00 & 0,00 & 0,50 \\
Llp & 0,33 & 0,00 & 0,33 & 0,66 & 0,66 & 0,66 \\
Rc & 0,00 & 0,00 & 1,00 & 1,00 & 1,00 & 1,00 \\
Dcbb & 1,00 & 1,00 & 0,50 & 0,50 & 0,50 & 0,50 \\
Wp & 1,00 & 1,00 & 0,50 & 0,50 & 0,50 & 0,50 \\
Qmsf & 0,00 & 0,00 & 0,00 & 0,66 & 1,00 & 0,00 \\
Irt & 0,00 & 0,00 & 0,00 & 0,00 & 0,00 & 1,00 \\
Pps & 0,33 & 0,33 & 0,33 & 1,00 & 1,00 & 1,00 \\
Fdb & 0,66 & 1,00 & 1,00 & 1,00 & 1,00 & 1,00
\end{tabular}

Primeira

\begin{tabular}{|c|c|c|c|c|c|c|}
\hline Olp & 0,00 & 0,00 & 0,00 & 0,00 & 0,00 & 0,05 \\
\hline LIp & 0,33 & 0,00 & 0,33 & 0,66 & 0,66 & 0,13 \\
\hline $\mathrm{Rc}$ & 0,00 & 0,00 & 1,00 & 1,00 & 1,00 & 0,10 \\
\hline $\mathrm{Fi}$ & 1,00 & 1,00 & 0,50 & 0,50 & 0,50 & 0,05 \\
\hline Ipt & 0,00 & 0,00 & 0,00 & 0,33 & 0,50 & 0,08 \\
\hline Pps & 0,33 & 0,33 & 0,33 & 1,00 & 1,00 & 0,15 \\
\hline $\mathrm{Fdb}$ & 0,66 & 1,00 & 1,00 & 1,00 & 1,00 & 0,20 \\
\hline
\end{tabular}

agregação

Segunda

agregação

\begin{tabular}{lllllll} 
ADIU & 0,33 & 0,33 & 0,45 & 0,64 & 0,67 & 0,74 \\
ADIW & 0,35 & 0,35 & 0,46 & 0,68 & 0,71 & 0,76 \\
\hline
\end{tabular}

Fonte: Até 1996 - Rigolon (1997), após esse período elaboração própria. 